\title{
Using a Laser Scanning to Construct a 3D Numerical Model to Study the Flooding Risk in Urban Area
}

\author{
R. E. Meouche, M. Rezoug, W. Vandevenne, and R. Hamzaoui
}

\begin{abstract}
Risk studies are very important to avoid human and economic losses. However the study cost becomes very expensive in some case when the site scale is very large. That's why; using a numerical simulation with a small-scale model is considered as a suitable solution. In order to have accurate results on which we can rely to assess the risk, it is necessary to use sophisticated means to simulate with a high efficiency the study area. One of the very accurate means able to do that is based on laser scanning techniques. This article describes how a 3D laser scanning can provide quickly and accurately a 3D model of the study area reduced model for the risk simulation step. Moreover, the necessary processing steps in the laser software named Geomagic v12, are highlighted to achieve a neat 3-D model. The article also describes the preparation of the model for the numerical simulation step.
\end{abstract}

Index Terms-3D laser scanner, processing data, 3D modeling, simulation.

\section{INTRODUCTION}

$3 \mathrm{D}$ laser scanning is the very newest invention related to applications for mapping software packages. The need for an inexpensive environmental analysis in a short time span has initiated the development of effective terrestrial laser scanning. Since 1990, this effective application has evolved quickly to compensate for the extensive photogrammetry.

The principle of a laser scanner is to collect spatial information, such as 3D coordinates. The environment of a 3D object becomes scanned, and the spatial data of millions of items that relate to the shape of the object, are collected in a few minutes. The result of a single scan is a point cloud in which each point has three parameters: a measured distance, and two angles.

The intention of this technique is to create digital files, such as 2D drawings or 3D models; the latter can be used for phenomenon simulations or to obtain sections or slices of such a model. The current technique has a wide range of application domains including the medical and industrial sectors, but it is most frequently used in civil engineering and land Surveying. Several researchers [1]-[5] have written articles about the field of 3D laser scanning.

Manuscript received December 18, 2012; revised May 20, 2012.

R. El Meouche, M. Rezoug, and R. Hamzaoui are with the Institute of Research on Constructibility- ESTP Paris, 28, avenue du Président Wilson 94234 Cachan cedex, France (e-mail: relmeouche@adm.estp.fr).

W. Vandevenne is with the Universety Hogent, Belgium.

\section{PRINCIPLES OF LASER SCANNING}

\section{A. Statistic or Dynamic}

The field of laser scanning technology can be split mainly into two parts: static and dynamic. The static definition includes terrestrial laser scanning, which has both a high precision and a relatively high point density. If the scanner is moved or carried along on a mobile platform while the measurements are being calculated and collected, then it falls under the dynamic definition. Mobile systems, such as a GPS (Global Positioning System), are used to determine the position of the scanner [6].

\section{B. Laser Scanner and the Electromagnetic Spectrum}

There are main three components by which the electromagnetic (EM) spectrum can be determined or estimated: energy, wavelength and frequency. The wavelength of the electromagnetic spectrum can range from short size (gamma radiation) to long size (radio waves). The human eye can only perceive a small portion of EM radiation. This part of the spectrum is called visible light, composed of the distribution of colors that can be observed after the dispersion of a light beam through a prism. The relationship between the working of a scanner and the EM waves can be found in a narrow part of the spectrum. A laser scanner generates a controlled low divergent, polarized beam with a specific wavelength $(1064 \mathrm{~nm}$ near infrared to green $532 \mathrm{~nm}$ ), called laser light. It is important to know that this area is divided into hazard classes, from $1 \mathrm{R}$ (non-hazardous) to 4 (where laser light can cause bodily injury) [7].

\section{Terrestrial Measurements Systems}

There are two types of measuring systems: active and passive. The passive measuring systems rely on the beams reflected and sent out by the environment. There is no laser emitter that generates beams, only a receiver. The active systems use a controlled beam and a receiver that analyzes the reflected waves. Two current systems are triangulation (based on Thales' geometric calculations) or time of flight (TOF).

In triangulation systems, the emitter and the receiver are separated by a given distance. This distance is one of the parameters necessary to define a triangle. The other two parameters are obtained by measuring the angles at which a beam is transmitted and received after reflection. From these three parameters one can obtain the distance between the object and the scanner (distance OS), which is given in micrometers [8].

Another technique is TOF, which is based on the fact that a 
laser beam needs a certain amount of time to reach the object, hit the object's surface, and then reflect and rebound. With a pulsed-based TOF system, the surface is scanned point by point at a speed of 2000 to 50000 points per second. This system does use rather pulses. A system clock keeps accurate the time interval between sending and receiving a pulse. By multiplying the time of flight with the speed of light, one obtains a distance: receiver-surface-transmitter. One must then divide this distance by two, to get the distance OS. Another TOF technology utilizes continuous wave. This is based on the measurement of phase difference. The emitter sends a continuous sinusoidal modulated wave, which has a fixed frequency and amplitude. The receiver collects a wave with a time delay. The sum of the number of complete wavelengths (given as an integer) and the phase difference, divided by two gives the distance OS. Both systems measure the distance OS with an accuracy in $\mathrm{mm}$ [6].

\section{Error Analysis}

Four types of errors can occur during the scanning process; instrumental, ambient, object-related and methodological errors. A good knowledge of the metrological aspects is useful to understand some results of the scanning process.

An instrumental related error could be a bad alignment of the measuring system for the rotation angle of the mirror or axes errors. Also some divergence can accur, such as increasing amplitude, during the propagation of a laser beam, as a wave moves along in a smooth oscillating pattern (sinuosity). Such divergence exerts an influence on both the resolution of the point cloud and on the uncertainty of the position of the central axis of the beam. Another instrumental related error is the "mixed edge" problem, which occurs when the beam splits in two once it hits the edge of an obstacle.

Environmental factors that may cause errors are: the temperature of the scanner, the atmosphere, the radiation interference and distortion.

Transparency, color characteristics, the angle of incidence with respect to the normal and the reflection properties of the surface to be scanned can be sources of object-related errors. In addition, the chosen measuring technique and the experience of the user could result in methodological errors, such as oversampling by setting a grid with a higher resolution than the scanner's capacity or an incorrect registration of the point clouds in the software [3], [4].

\section{LASER SCANNER APPLICATIONS}

After the recreation of a possible flooding area performed on a reduced model (small scale model such as $1 \mathrm{~m} \mathrm{x} 2 \mathrm{~m}$, see Fig. 1), a digital model is necessary for the implementation of numerical simulations. Data acquisition is the first step of the digitization process. Once the dataset or point cloud from each of the four scans is obtained, the data is compiled altogether into one complete point cloud, the registration step. The final step is point cloud processing, a time-consuming task that is done manually. This includes: representations, data improvement (noise filtering, resampling, hole filling) and meshing (the generation of triangles between the points). The result should be a neat, meticulously accurate, digital 3D model that can then be integrated into a simulation program, such as ANSYS Fluent.

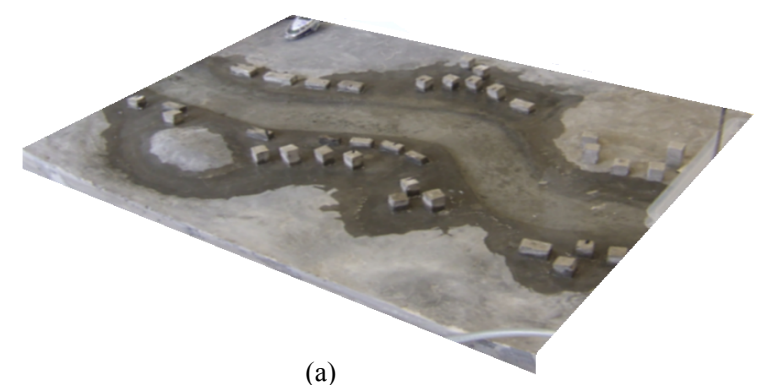

(a)

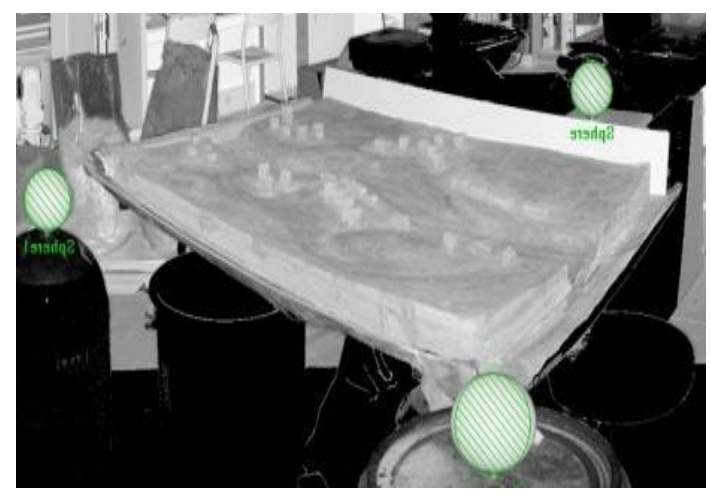

(b)

Fig. 1. Application of a laser scanning on a real small scale model (a) real model, (b) a global point cloud.

\section{A. Processing and Software}

Four scans of the model are taken with a phase-based laser scanner: Faro Focus 3D (data acquisition). Each scan contains a scan time of 10 minutes and delivers a $120 \mathrm{Mb}$ file. The scans contain a separate dataset that will be loaded into the Geomagic 12 software packet. Unnecessary scanned surfaces will be first removed for further processing. This is necessary for the creation of a model and the performance properties of the computer. The first manual registration is to identify corresponding points between each scan. Each scan having a different spatial orientation will be put together in one coordinate system. The four point clouds now form a completed model, although there is not yet a perfect alignment between the point clouds. For the global alignment, an advanced mathematical algorithm will be used. Now the point clouds are ready to be assembled in 1 object. Another important step is to create polygons and filling holes. The creation of a surface is the last step for a 3D model that can be exported for simulation (See Fig. 2). Other processing software packets are FARO Scene and FARO Cloud.

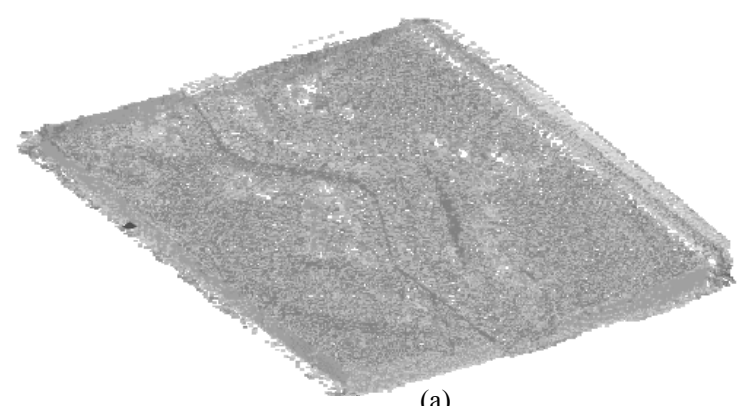

(a) 


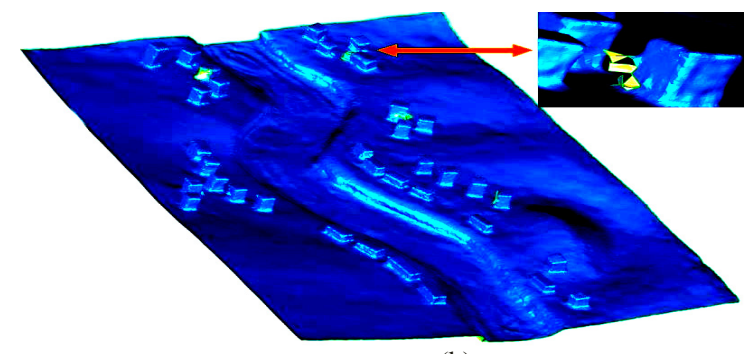

(b)

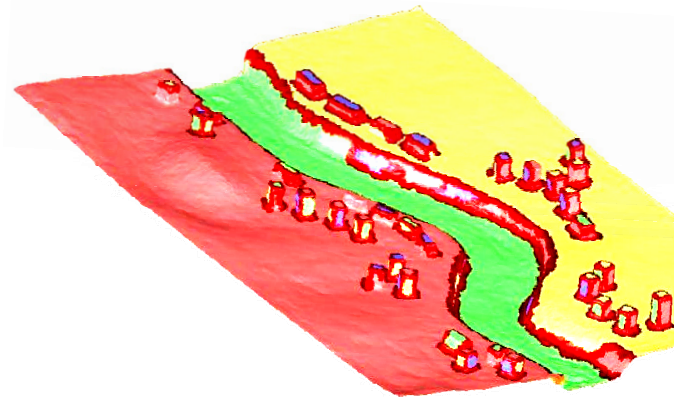

(c)

Fig. 2. Processing steps. (a) Point clouds; (b) create polygons and filling holes; (c) creation of a surface.

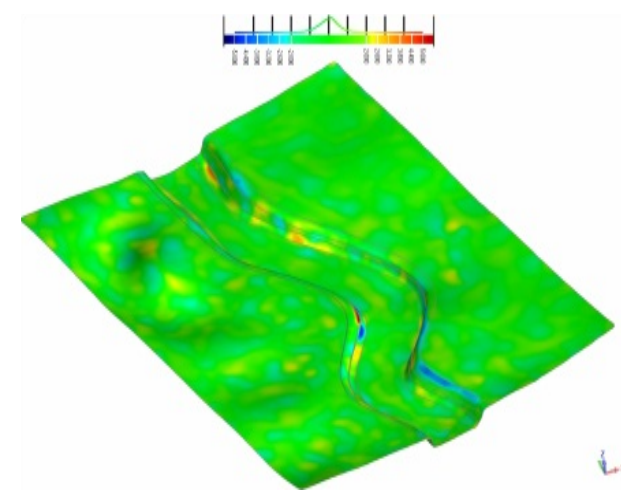

(a)
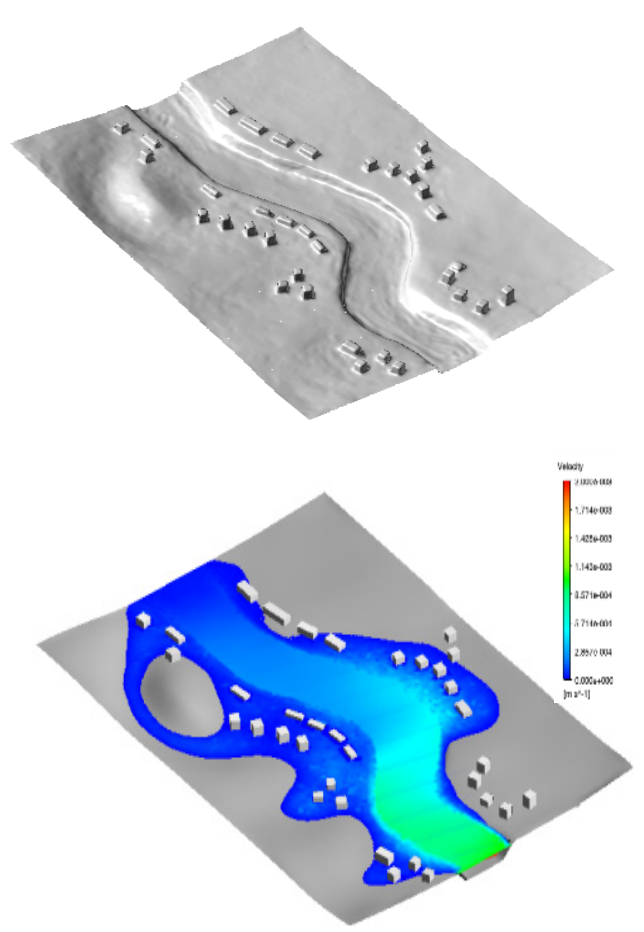

(c)

Fig. 3. Use of the 3D Laser scanning model in the simulation study. (a) Surface model; (b) Global model; (c) flood simulation.

\section{B. Simulation}

The risk and impact of floods in urban areas has been increased over the last few decades as population and urbanization processes rapidly increase and subsequently more and more people and properties are being concentrated in flood-prone coastal zones and river flood-plains. Floods in urban areas can be much more devastating than in any other areas. They are responsible for more than $50 \%$ of the fatalities and for about $30 \%$ of the economic losses [1], [2]. That's why development of modeling methods able to represent accurately and effectively the flood propagation event in urban areas allows a better prediction and risk assessment, in order to avoid and limit the human and economic losses. New data collection techniques have emerged which alleviate the traditional problem of lack of data for topographic and terrain modelling. In addition, numerical techniques have matured, providing robustness and efficiency in model simulation. Since the 1990s, especially in recent years, the use of geographic information systems (GIS) technology and its powerful functions of spatial analysis and 3D visualization to simulate and display flood area and to evaluate disaster damage has become a research hotspot [10], [11]. Laser scan 3D is one of the most effective techniques able to represent a 3D urban area with very high accuracy. In this example study a 3D model of urban area (reduced model) crossed by an urban river. After having treated the scanned data of the reduced model with a set of CAO and Software for the Laser Lake; Geomagic, FARO Scene, AutoCAD, a CFD (computational fluid mechanics) simulation of the flooding is implemented with this model to give accurate results close to reality [12].

\section{CONCLUSION}

To create a digital model for computer simulations, a 3D laser scanner can be used for the integration of a small-scale model into specific software. A good knowledge of the principles of the laser scanning is important to better understand the laser working and improve scanning results. The data acquisition knows the highest degree of automation in the 3D point cloud processing. Once the scans are compiled all together (the registration), the complete point cloud has to be improved and meshed. These steps can be very costly and time-consuming, because they must be done manually. Geomagic V12 is one of the best software destined for processing of 3D points cloud. Programs like FARO Scene and FARO Cloud do also allow 3D point cloud improvement; however, they are less appropriate to create 3D models for simulation problems. Scanned and processed data can be the main input data for numerical simulation models such as the CFD simulation for the case of urban flooding, the example of this study.

\section{REFERENCES}

[1] Education and culture Leonardo da Vinci, Theory and Practice on Terrestrial Laser Scanning, pp. 241, 2008.

[2] S. Berger, Etude de la modélisation d'une reduced model 3D et de l'intégration de données, pp. 84, 2011.

[3] T. Landes, Confrontation de la lasergrammétrie aux techniques de relevé conventionnelles et développement d'outils numériques pour la restitution architecturale, pp. 6, 2008 . 
[4] W. Boehler, Investigating Laser Scanner Accuracy, pp. 19, 2003.

[5] Y. J. Hyun, Analyse et conception de scanners laser mobiles dédiés à la cartographie 3D d'environnements urbains, pp. 132, 2011.

[6] F. Brosed, 3D Geometrical Inspection of Complex Geometry Parts Using a Novel Laser Triangulation Sensor and a Robot, pp. 21, 2010.

[7] T. Landes and P. Grussenmeyer, Les principes fondamentaux de la lasergrammétrie terrestre, AFT-XYZ, no. 128, pp. 37-49, 2011.

[8] K. Blyth, "A Telenetwork for Acquisition, Processing and Dissemination of Earth Observation Data for Monitoring and Emergency Management of Floods," Hydrological Processes, vol. 11, pp. 1359-1375, 1997.

[9] M. Pelling, "Assessing Urban Vulnerability and Social Adaptation to Risk: A Case Study from Santo Domingo," International Development Planning Review, vol. 24, no. 1, pp. 59-76, 2002.

[10] J. Bender, D. Finkenzeller, and P. Oel, "HW3D: A tool for interactive real-time 3D visualization in GIS supported flood modeling," in Proc. of the 17th International Conference on Computer Animation \& Social Agents, Geneva, Switzerland, July, 2004.

[11] C. Wang, T. R. Wan, and I. J. Palmer, "A real-time dynamic simulation scheme for large-scale flood hazard using 3D real world data," in Proc. 11th International Conference on Information Visualisation, Zürich, Switzerland, pp. 607-612, 2007.

[12] M. B. Abbott and D. R. Basco, Computational Fluid Dynamics: An Introduction for Engineers, New York: John Wiley, 1989.

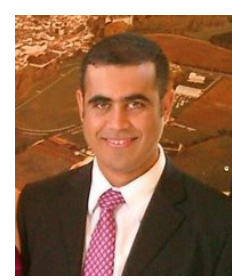

Rani El Meouche is an associate professor in the Constructibility Research Institute (ESTP/IRCFrance), and $\mathrm{He}$ received Master's Degree in Engineering/Surveying from ESGT - LE MANS, France, M.S.c in Geographical Information Sciences from University of Marne La Vallée, France, and Ph.D. degree from the same university in 2007.

His principal research interests lie in the field of Geomatics/Surveying Engineering, 3D Modeling, BIM, GIS and GNSS.
Currently he is the head of the department of surveying and Geomatics in ESTP.

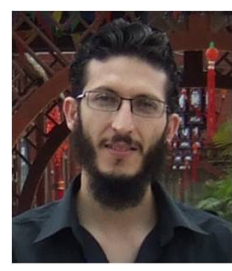

Mehdi Rezoug is an assistant professor in the Constructibility Research Institute (ESTP/IRCFrance); his principal research interests lie in the field of Multicriteria Optimization and Multiphysics Modeling in mechanics and civil engineering.

For his $\mathrm{PhD}$, he developed a numerical method to construct a three-dimensional model of complex urban areas using specific programming methods combined with GIS data and CAD software in order to study different flooding scenarios. A great part of his thesis was also interested to explore the different solutions and spatial planning projects to be made in urban environments in order to reduce the risk caused by the floods. In this context, a multi-criteria optimization approach based on evolutionary algorithms has been developed and coupled with the preparametered CFD modeling in order to find the optimal configuration of the city representing the least risk and the maximum comfort.

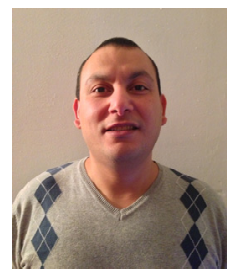

Rabah Hamzaoui is researcher scientist from IRC/ESTP Cachan France. He has professor grade (HDR). I have worked in nanomaterials obtained by mechanical alloying and their properties (magnetic, mechanic, structure....) since 1998. Actually, He is interested by construction materials (concrete, mortar, soils,...). His main objective is to introduce Nanomaterials in construction materials in order to propose smart construction materials.

W. Vandevenne is a student Faculty of Applied Engineering Sciences, University College Ghent. 